Schizophrenia. London: Independent Working Party.

LITTLEJOHNS, P., CLUZEAN, F., BALE,

original

papers of clinical practice guidelines for the
R., et al (1999) The quantity and quality management of depression in primary care in the UK. British Journal of General Practice, 49, 205-210.

LIVINGSTONE, M. MACKAY, A KENDAL, R., et al (1995) Services for PeopleAffected by Schizophrenia.

Edinburgh: CRAG/SCOTMEG Working

Group on Mental Illness.

NATIONAL INSTITUTE FOR CLINICAL

EXCELLENCE (NICE) (2002) Ful

guidance on the use of newer (atypical) antipsychotics for the treatment of schizophrenia. TechnologyAppraisal No. 43

ROYAL COLLEGE OF PSYCHIATRISTS' RESEARCH UNIT (2000)

Guidelines in Mental Health. London:

The Royal College of Psychiatrists' Research Unit.

ROYAL COLLEGE OF PSYCHIATRISTS, BRITISH PSYCHOLOGICAL SOCIETY AND UNIVERSITY OF YORK (1999) The Management of Schizophrenia Part 1 : Pharmacological Treatments. London: Royal College of Psychiatrists, British Psychological Society and University of York. AUDIT GROUP (SCRAG) (1993)

Clinical Guidelines - A Report by a Working Group. Edinburgh: The

Scottish Office.

SCOTTISH INTERCOLLEGIATE GUIDELINES NETWORK (1998)

Psychological Interventions in the Management of Schizophrenia.

Edinburgh: Scottish Intercollegiate

Guidelines Network.
SCOTTISH CLINICAL RESOURCE AND

TEES AND NORTH EAST YORKSHIRE NHS TRUSTS DRUG AND THERAPY COMMITTEE (2000) Prescribing and Shared Care Guideline for

Schizophrenia. Durham: Tees and North East Yorkshire NHS Trusts Drug and Therapy Committee.

WORLD HEALTH ORGANIZATION COLLABORATING CENTRE FOR RESEARCHAND TRAINING FOR MENTAL HEALTH (2000) WHO Guide to Menta Health in Primary Care. London: Royal Society of Medicine Press.

Helen Snowden Senior ProjectWorker ～*Sarah Marriott Consultant Psychiatrist, Paterson Centre for Mental Health, 20 South Wharf Road, London W2 1PD

DAVID MEAGHER AND MARIA MORAN

\title{
Sub-optimal prescribing in an adult community mental health service: prevalence and determinants
}

\author{
AIMS AND METHOD \\ To compare prescribing practice in a \\ community mental health service \\ with evidence-based guidelines and \\ identify factors related to \\ sub-optimal prescribing. All current \\ patients ( $n=640$ ) were assessed \\ regarding six key aspects of \\ prescribing (polypharmacy, \\ high-dose treatment, use of \\ thioridazine/maintenance \\ benzodiazepine/maintenance \\ hypnotic or routine anticholinergic \\ treatment). The relationship of \\ quality of prescribing practice to \\ demographic, illness and service \\ variables was examined by regression \\ analysis.
}

\author{
RESULTS \\ Five-hundred and five (79\%) patients \\ were receiving psychotropic \\ medication. Of these, 232 (46\%) had \\ evidence of sub-optimal prescribing \\ practice. Mean prescribing practice \\ quality score was $0.75 \pm 0.99$. \\ Maintenance benzodiazepine/ \\ hypnotic (31\%) and anticholinergic \\ $(30 \%)$ use were particularly common. \\ Prescribing practice quality score was \\ higher in those receiving depot anti- \\ psychotic treatment $(P<0.01)$ and in \\ older patients $(P<0.01)$. Scores were \\ significantly lower in patients whose \\ principal medical contacts were with \\ a consultant rather than a junior \\ doctor $(P<0.001)$.
}

\begin{abstract}
CLINICAL IMPLICATIONS
Prescribing practices in real-world settings frequently deviate from evidence-based guidelines. The quality of prescribing is related to patient, illness and service variables. In particular, greater contact with consultant staff is linked to better practices. Patients receiving depot antipsychotics are especially liable to less judicious prescribing practice.
\end{abstract}

Although guidelines for optimal use of psychotropics are widely available, prescribing in real-world settings routinely differs from suggested standards. Polypharmacy, for example, is strongly discouraged in treatment guidelines, but typically found in $25 \%$ of out-patient attendees (Stahl, 2000). In recent years, two large cross-sectional studies of antipsychotic prescribing across mental health services within the UK indicated that approximately $50 \%$ of in-patients are in receipt of more than one antipsychotic agent (Lelliott et al, 2002; Harrington et al, 2002b).

Other aspects of psychotropic prescribing are repeatedly highlighted. High-dose antipsychotic use is not supported by evidence of clinical efficacy and is linked to adverse effects, including a risk of sudden death (Mackay, 1994). High doses should only be used after consideration of alternative approaches (e.g. switching to clozapine therapy) and discontinued if not of obvious benefit at 3 months (British National Formulary, 2002). More recently, thioridazine use has been restricted to second-line treatment of psychosis, due to evidence of cardiotoxicity potential (Reilly et al, 2000). Moreover, given that it may be subject to further restrictions or discontinuation, it is prudent to minimise its use.

Anticholinergic agents alleviate neuroleptic-induced extrapyramidal side-effects, but have adverse cognitive effects, alter absorption of other oral medications and have abuse potential (Marken et al, 1996). Short-term, as-required use prevents extrapyramidal side-effects in the vast majority of patients (World Health Organization, 1990; Steele et al, 2000), but routine use is nonetheless commonplace (Kelly et al, 1998). 
Benzodiazepine and hypnotic agent use should be restricted to short-term treatment of severe and disabling anxiety or insomnia (British National Formulary, 2002). More prolonged use is associated with reduced motor performance, cognitive impairments, paradoxical effects and dependence. As a consequence, treatment for greater than 4 weeks is not recommended (Department of Health and Children, 2002).

The implementation of agreed prescribing guidelines is increasingly used as an effective way of assuring quality in drug treatment of major mental disorders (Steele et al, 2000; Taylor et al, 2000). In this study, we compare current practices within a generic multidisciplinary service to suggested guidelines and experiences elsewhere. Predictors of the quality of current prescribing are also examined.

\section{Methods}

The St Anne's service provides mental health care for a mixed rural/urban population of 46000 in Southeast City and County Limerick. A generic multidisciplinary team operates from a community-based day hospital, providing a range of psychological and pharmacological treatments.

Over 3 days between July and September 2001, all open casefiles in the service were evaluated with regard to demographics, history of service contact and current treatment (including drug treatment and multidisciplinary team member contacts). Clinical diagnoses were made by the consultant psychiatrist or a senior registrar in psychiatry according to ICD-10 criteria (World Health Organization, 1993).

\section{Assessment of prescribing practices}

The quality of prescribing practices within the service was compared with standards outlined in the Psychotropic Drug Directory (Bazire, 2001) and the Maudsley Prescribing Guidelines (Taylor et al, 2001). Six key aspects of prescribing thought to represent sub-optimal prescribing practice were rated (absent $=0$, present $=1$ ) to produce a prescribing practice quality score, such that high scores reflect less judicious prescribing practice (see Box 1). Patients were considered in receipt of treatments

\section{Box 1. Prescribing practice quality items and scoring}

Polypharmacy i.e. use of two agents of same class (1 point)

Receiving thioridazine (1 point)

Use of high-dose medication i.e. total dose in chlorpromazine equivalents greater than 1000 mg per day (1 point)

Maintenance benzodiazepine treatment (1 point)

Maintenance hypnotic treatment (1 point)

Routine use of anticholinergic agent (1 point)

(Scores range 0-6; higher scores suggest less optimal prescribing) if they were receiving a regular prescription for the agent at the time of the audit or had received the agent on an as-required basis for more than $50 \%$ of the previous 4 weeks. Chlorpromazine equivalents were calculated according to criteria outlined by Centorrino et al (2002). The total score was considered an indicator of quality of overall prescribing and its relationship to other aspects of service use was examined.

\section{Statistical analysis}

The statistical analyses were conducted using SPSS Version 10.1. All significance tests were two-tailed. Chisquared statistics were used to compare proportions of those attending consultant or junior medical staff with serious mental illness or other mental disorders. The mean age of those receiving drug treatments was compared with independent samples $t$-testing. The differences in mean scores were calculated with Mann-Whitney $\mathrm{U}$ tests. Independent predictors of prescribing practice quality score were identified by linear regression modelling.

\section{Results}

\section{Demographics}

A total of 640 case files were identified as currently open. Slightly more than half (51\%) were female. The mean age of the overall population was $41 \pm 14$ years. The consultant was the principal medical contact for $37 \%$ of the caseload $(n=238)$. Greater consultant involvement was evident in those patients with more severe forms of mental illness, such as psychosis or bipolar disorder $(P<0.001)$.

Five-hundred and five (79\%) patients were receiving psychotropic agents. The frequency of sub-optimal prescribing practices highlighted for examination are depicted in Table 1. Overall, 232 (46\%) patients had at least one aspect of prescribing that could ideally be improved.

\section{Antipsychotic use}

Two-hundred and seventeen patients were receiving antipsychotics; 169 oral agents and 71 long-acting intramuscular agents. Of oral agents prescribed, 92 patients were receiving conventional antipsychotics

\section{Table 1. Prescribing practices $(n=453)$}

\begin{tabular}{lc} 
Practice & $n$ \\
\hline Antipsychotic polypharmacy & 49 \\
Antidepressant polypharmacy & 11 \\
Thioridazine treatment & 36 \\
High dose psychotropic use & 10 \\
Maintenance benzodiazepine treatment & 78 \\
Maintenance hypnotic treatment & 106 \\
Routine use of anticholinergic agent & 67 \\
Depot frequency at greater than suggested guidelines & 4
\end{tabular}

original papers 
(chlorpromazine, thioridazine, haloperidol, trifluoperazine) and 97 newer agents (risperidone, olanzapine,

original papers receiving thioridazine. clozapine, sulpiride, amisulpiride). Thirty-six patients were

The mean dose of antipsychotic treatment in chlorpromazine equivalents per day was $345 \pm 324 \mathrm{mg}$ (range 25-2225 mg per day). Ten patients (4.9\%) were receiving in excess of $1000 \mathrm{mg}$ of chlorpromazine equivalents per day, all of whom were subject to antipsychotic polypharmacy. Antipsychotic polypharmacy was evident in 49 (23\%) patients; 21 patients were in receipt of two or more oral antipsychotics and 34 patients were receiving combined oral/intramuscular agents. Fifteen patients were receiving oral atypical agents as well as a depot antipsychotic.

Seventy-one patients were receiving intramuscular antipsychotic preparations. These patients were receiving the equivalent of $172 \mathrm{mg}$ chlorpromazine per day via intramuscular preparation - much lower than the mean dose for the overall population receiving antipsychotics (345 mg per day), but when the total dose of antipsychotic (oral+intramuscular) was calculated, these patients were in receipt of higher mean doses $(375 \pm 386 \mathrm{mg})$ than patients exclusively receiving oral antipsychotics. Four patients (5\%) were receiving depot preparations more frequently than suggested guidelines.

The pattern of antipsychotic prescribing varied considerably according to whether patients were predominantly in contact with a junior doctor or a Consultant. Consultant contact was linked to greater use of atypical antipsychotics and less use of thioridazine (see Table 2). A higher percentage of patients in the 16-35 age group were receiving atypical agents (76\%) compared to the over-35 age group (49\%).

\section{Anticholinergic agents}

Sixty-seven patients within the service were in receipt of maintenance anticholinergic therapy - almost a third $(30 \%)$ of those receiving antipsychotic medication. Thirty-nine of these patients were receiving depot antipsychotics (i.e. $54 \%$ of those on depot preparations) and 28 were on oral antipsychotic preparations only (16\% of those receiving exclusively oral medications).

Table 2. Antipsychotic prescribing according to principal medical contact

\begin{tabular}{lccc} 
Antipsychotic & $\begin{array}{l}\text { Consultant } \\
(n)\end{array}$ & $\begin{array}{l}\text { Junior doctor } \\
(n)\end{array}$ & Total (\%) \\
\hline Risperidone & 29 & 15 & $44(20 \%)$ \\
Olanzapine & 24 & 15 & $39(18 \%)$ \\
Clozapine & 6 & 2 & $8(4 \%)$ \\
Sulpiride & 1 & 1 & $2(1 \%)$ \\
Amisulpiride & 1 & 0 & $1(0.5 \%)$ \\
Typical & 35 & 52 & $87(40 \%)$ \\
Thioridazine & 10 & 26 & $36(16 \%)$ \\
Total & 106 & 111 & 217 \\
\hline
\end{tabular}

Table 3. Relationship between mean prescribing practice

quality (PPQ) score and diagnosis

\begin{tabular}{lrl} 
Diagnosis & $n$ & $\begin{array}{l}\text { Mean PPQ score } \\
(95 \% \mathrm{CI})\end{array}$ \\
\hline Psychosis & 160 & $1.11(0.92-1.30)$ \\
Bipolar anxiety disorder & 41 & $0.80(0.52-1.09)$ \\
Recurrent depressive disorder & 207 & $0.59(0.48-0.71)$ \\
Substance abuse disorder & 28 & $0.11(0.05-0.27)$ \\
Anxiety disorder & 37 & $0.57(0.35-0.78)$ \\
Other disorder & 29 & $0.55(0.15-0.95)$ \\
& &
\end{tabular}

Table 4. Stepwise regression model for prediction of

prescribing practice quality (PPQ)

\begin{tabular}{lll}
\hline Factor & $P$ & $R^{2}$ \\
\hline Receiving depot antipsychotic & $<0.01$ & 0.15 \\
Age & $<0.01$ & 0.17
\end{tabular}

\section{Benzodiazepine/hypnotic use}

Almost one-in-four patients attending the service (24\%) were receiving benzodiazepine anxiolytic or hypnotic agents. Similar rates of benzodiazepine prescribing were found where the principal medical contact was with the Consultant $(26 \%)$ or junior doctor $(28 \%)$. Similar rates were evident across diagnostic boundaries, with the notable exception of patients with substance misuse problems.

\section{Prescribing practice quality scores}

The mean score for the total population was $0.75 \pm 0.99$. Of these, 232 (45.9\%) of the patients receiving psychotropics had at least one aspect of prescribing that was considered sub-optimal. Mean scores were higher in men $(0.86 \pm 1.07)$ compared to women $(0.64 \pm 0.89)(P=0.01)$; those patients whose principal medical contact was with the junior doctor $(0.89 \pm 1.05)$ compared to the consultant $(0.62 \pm 0.92)(P<0.001)$; and those patients receiving intramuscular antipsychotics $(1.63 \pm 1.29)$ compared to those receiving oral antipsychotics $(0.94 \pm 0.97)(P<0.001)$. Table 3 depicts the relationship between mean score and diagnosis. Higher scores were evident in those with psychotic illness.

Linear regression analysis was performed to identify factors that significantly predicted prescribing practice quality score independent of the effect of other variables (see Table 4). Two variables independently predicted the score; being in receipt of an intramuscular antipsychotic and older age were associated with higher scores.

\section{Discussion}

This cross-sectional study of prescribing in the real-world setting of a busy generic multidisciplinary mental health service indicates that, in keeping with studies elsewhere, prescribing practices frequently deviate from recommended 
evidence-based guidelines. Almost half of our population had one or more aspect of their medication regime outside suggested guidelines. This can reflect the value of unproven prescribing strategies, but might also reflect sub-optimal practice due to educational deficits or other constraints. Recent studies point towards the latter and suggest that antipsychotic polypharmacy, for example, frequently reflects non-adherence to suggested guidelines around the adequate duration of a therapeutic trial or guidelines for clozapine use (Taylor, 2002).

The levels of antipsychotic polypharmacy are typical of community settings (Stahl, 2000). High-dose antipsychotic use was relatively infrequent. Intramuscular antipsychotic doses were relatively low and might relate to the high rates of supplementation with oral antipsychotic agents. Routine prescribing of anticholinergic medications was particularly common in those receiving intramuscular antipsychotic preparations. In view of these findings, and the less overall judicious prescribing in patients receiving intramuscular agents, this group has been prioritised for medication management in the coming year.

Recent evidence around potential cardiotoxicity has resulted in markedly reduced use of thioridazine. In the St Anne's service, continued use has occurred in selected cases after consideration of relative risks and benefits. It is interesting that patients with greater consultant contact are less likely to continue thioridazine use. This finding, and the more frequent use of atypical antipsychotics, suggests greater ability to effect antipsychotic switches in consultant staff.

Many patients were receiving maintenance benzodiazepine agents, either as anxiolytics or hypnotics. Similar rates were evident regardless of the principal medical contact, raising the issue of the origin of this prescribing. In view of recent debate around the use of benzodiazepines in primary care (O'Callaghan, 2001; Department of Health and Children, 2002), we have elected to study benzodiazepine use in referrals from primary care to establish to what extent this problem is inherited. Similar rates of benzodiazepine use were evident across diagnostic boundaries with the exception of patients with substance misuse problems. This may reflect the overt recognition that benzodiazepine use can be inappropriate in this population, and indicates that benzodiazepine use can be restricted in particular populations.

It is reassuring that consultant contacts were concentrated on more severe mental illness. Moreover, consultant contact was associated with better overall prescribing practice, supporting the argument for consultant-driven services. Recent studies of antipsychotic prescribing habits in the UK have identified a range of patient and service variables (age, gender, Mental Health Act 1983 status, diagnosis and treatment setting) related to polypharmacy and high-dose antipsychotic use, but did not study clinician variables (Lelliott et al, 2002; Harrington et al, 2002a; 2002b). The higher prescribing practice quality scores evident in those with psychotic disorders reflect, in part, the emphasis on quality of antipsychotic prescribing in the scores, whereby four items directly relate to either antipsychotic or anticholinergic use. However, it is interesting that the relative quality of prescribing was less optimal in those receiving intramuscular rather than exclusively oral antipsychotics.

Service users frequently express a preference for continuity of contact with team members. This can result in compartmentalisation of the service, with a subpopulation having relatively little contact with senior medical staff. While this is acceptable if based on relative needs, it is important to ensure equity of service for vulnerable patient groups that may not advocate as well for themselves. One alternative is to rotate medical contacts so that available time with the consultant psychiatrist is spread out across the total caseload, with all service users receiving a regular review rather than focusing consultant contact on a particular sub-group. Historically, the St Anne's sector arrangements favoured consistency of medical contacts over rotation. As a consequence of this study, the out-patient clinic format has been altered towards rotation of medical contacts and particular populations. For example, those receiving intramuscular antipsychotics have been highlighted for more regular formal review by senior medical staff. The rationale behind these changes has been explained to patients and their carers, and the vast majority have been happy to cooperate with this approach to appointments.

The introduction of clear prescribing policies can significantly improve prescribing practice (Steele et al, 2000; Taylor et al, 2000). However, in other studies, education around guidelines produced only modest and transient improvement (Eagles et al, 2000). Identification of sub-optimal practices, along with clear guidelines as to how improvements can be made, is helpful for junior doctors who may lack knowledge around good practices. Moreover, given that decisions about prescribing are often greatly influenced by factors such as the attitudes of nursing staff or relatives, clear policies boost confidence to adhere to good practices. At the very least, regular review of prescribing acts as a disincentive to the commencement of new bad practices.

\section{Acknowledgements}

Dr Jean Saunders, Department of Biostatistics, University of Limerick. The Community Mental Health Team at St Annes, all of whom contributed to data collection and analysis.

\section{Declaration of interest}

None.

\section{References}

BAZIRE, S. (2001) Psychotropic Drug Directory. The Professionals Pocket Handbook and Aide Memoire. Bath: Bath Press.

CENTORRINO, P., EAKIN, M., BAHK,W. M., et al (2002) Inpatient antipsychotic drug use in 1998, 1993 and 1989
American Journal of Psychiatry, 159 1932-1935.

DEPARTMENT OF HEALTH AND CHILDREN (Republic of Ireland) (2002) Report of the Benzodiazepine Committee. Dublin: Government Publications. original papers 


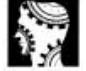

original papers
EAGLES, J. M., MCCANN, I., MACLEOD, N. et al (2000) Lithium monitoring before and after the distribution of clinical practice guidelines. Acta Psychiatrica Scandinavica, 101, 349-353.

HARRINGTON, M., LELLIOTT, P., PATON, C. et al (2002a) The results of a multicentre audit of the prescribing of antipsychotic drugs for in-patients in the UK. Psychiatric Bulletin, 26, $414-418$.

- - ctal (2002b) Variation between services in polypharmacy and combined high dose of antipsychotic drugs prescribed for in-patients. Psychiatric Bulletin, 26, 418-421.

KELYY C MCCREADLE, R G MACEWEN,T., et al (1998) Nithsdale schizophrenia surveys 17: fifteen year review. British Journal of Psychiatry, $172,513-518$
LELLIOTT, P., PATON, C., HARRINGTON, $M$. et al (2002) The influence of patient variables on polypharmacy and combined high dose of antipsychotic drugs prescribed for in-patients. Psychiatric Bulletin, 26, 411-414. MACKAY, A. V. P. (1994) High-dose antipsychotic medication. Advances in PsychiatricTreatment, 1, 16-23.

MARKEN, P. A., STONER, S. C., BUNKER, M. T. (1996) Anticholinergic drug abuse and misuse. CNS Drugs, 5, 190 -199.

O'CALLAGHAN, R. (2001) Irish doctors fight plans to curbValium. SundayTimes (news section), Nov 25.

REILLY, J. G., AVIS, S. A., FERRIER, I. N., et al (2000) QTc-interval abnormalities and psychotropic drug therapy in psychiatric patients. Lancet, 355 1048-1052.
ROYAL PHARMACEUTICAL SOCIETY OF GREAT BRITAIN (2002) British Nationa Formulary.

STAHL, S. (2000) Essential

Psychopharmacology. Neuroscientific Basis and Practical Applications, 2nd ed. Cambridge: Cambridge University

STEELE, J., DUNCAN, J. \& SHORT, A. (2000) An audit of anti-muscarinic drug-use at the state hospital. Psychiatric Bulletin, 24, 61-64.

TAYLOR, D. (2002) Antipsychotic prescribing - time to review practice. Psychiatric Bulletin, 26, 401-402.

Department of Adult Psychiatry, St Anne's Day Hospital, Roxboro Road, Limerick, Ireland, Maria Moran Senior Registrar in Psychiatry

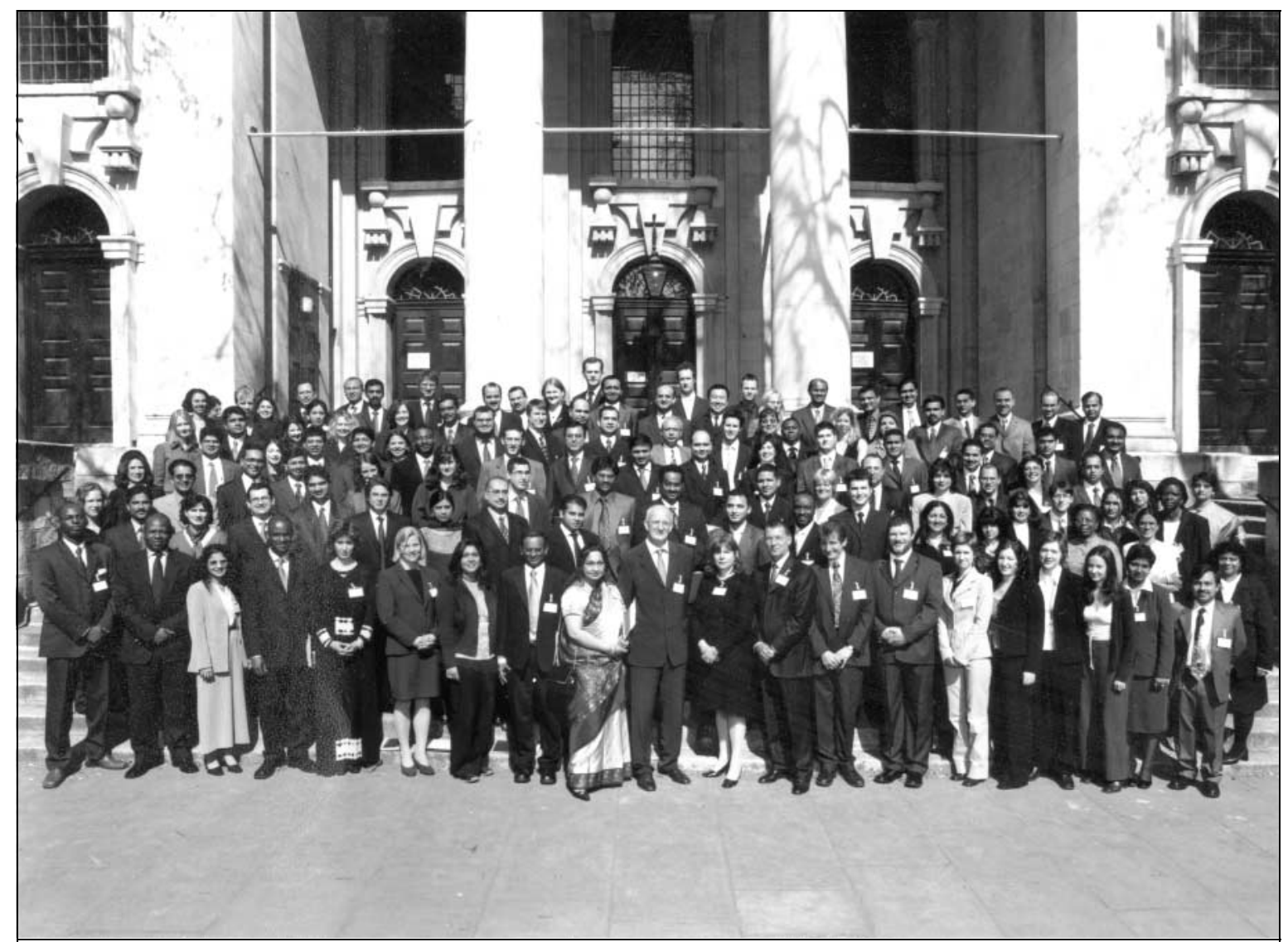

Group photograph from the new members' reception held at St John's, Smith Square, Westminster on 4 April 2003

If any new members have not yet ordered a copy of any photographs from this event and would like to do so, please contact

Dominic Turner, 45 Charlotte Road, London EC2 3PD, Tel/fax: 02077290122 